\title{
Leaders
}

\section{Recent advances in muscular dystrophies and myopathies}

\begin{abstract}
J R Anderson
\section{Introduction}

The era of histochemistry provided initial insight into skeletal muscle pathology, but over the past decade the tools of molecular genetics have dramatically accelerated the pace of discovery and elucidated the pathogenesis of many neuromuscular diseases.
\end{abstract}

\section{Muscular dystrophies DYSTROPHIN AND DYSTROPHIN ASSOCIATED PROTEINS}

Cloning of the gene responsible for Duchenne muscular dystrophy (DMD) on the short arm of the $\mathrm{X}$ chromosome in $1986^{1}$ was a major step that lead rapidly to identification of the gene product dystrophin by reverse genetics. ${ }^{2}$ Subsequently, investigation of the normal function of dystrophin revealed several dystrophin associated proteins and their roles in DMD, and other forms of muscular dystrophy are now being exposed.

Dystrophin is a long rod shaped protein that binds to actin at its $\mathrm{N}$ terminal and to a cell membrane associated glycoprotein, $\beta$-dystroglycan, at its $\mathrm{C}$ terminal domains (cysteine rich and carboxy terminal domains). $\beta$-dystroglycan is a $43 \mathrm{kDa}$ component of a large glycoprotein complex that spans the cell membrane and binds to laminin in the muscle cell basement membrane. Dystrophin therefore provides a link between the contractile cytoskeleton and the cell membrane which in turn is anchored to the extracellular scaffold (fig 1). ${ }^{34} \mathrm{~A}$ regular rib-like arrangement of bands of dystrophin encircling the muscle fibre conveys strength and flexibility to the cell membrane. ${ }^{5}$ In $\mathrm{DMD}$ dystrophin is absent or barely detectable and the cell membrane is unable to withstand muscular contractions. ${ }^{6}$ Tiny breaks develop in the membrane, permitting influx of calcium ions that trigger segmental hypercontraction, evident microscopically as large eosinophilic opaque fibres, and activate proteases ultimately leading to muscle fibre necrosis. Most patients (60-70\%) with DMD have a detectable deletion or duplication in the dystrophin gene; two thirds of these cases are

\section{Accepted for publication \\ 3 November 1994 \\ Histopathology, Box \\ 235, Addenbrooke's \\ Hospital, Hills Road, \\ Cambridge CB2 2QQ}

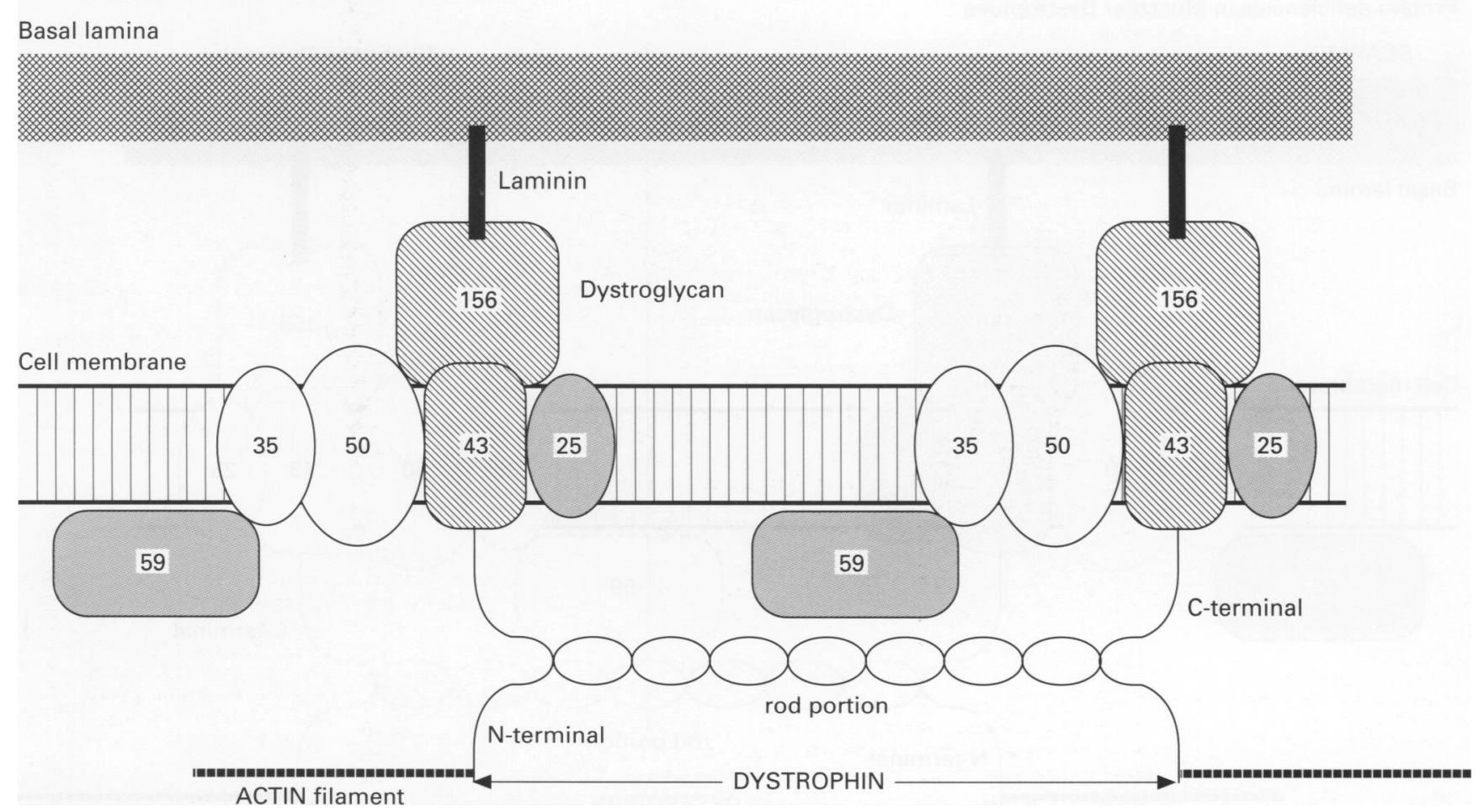

Figure 1 Dystroglycan $=(\alpha) 156 D A G+(\beta) 43 D A G ;$ Adhalin $=50 D A G$ closely linked to $35 D A G ; 25 D A P($ dystrophin associated protein $)=$ transmembrane protein; 59DAP = cytoskeletal protein (numbers refer to $\mathrm{kDa}$ value). 
familial, one third due to new mutations. Most deletions occur in one of two hot spots in the gene that can be rapidly detected by polymerase chain reaction (PCR) on peripheral blood cells. Likewise, detection of obligate carrier females and prenatal diagnosis is possible. ${ }^{7}$ Becker dystrophy is also caused by a dystrophin mutation, but the protein is present in a truncated form and the phenotype is correspondingly milder. The differences between the Duchenne and Becker phenotype are largely explained by the frameshift hypothesis. ${ }^{8}$ In DMD, as a result of the mutation, the distal reading frame conveys a nonsense message and only sequences upstream can be translated giving a small unstable molecule, whereas in Becker dystrophy intact codons are deleted and the open reading frame is maintained. A shorter protein is translated but provided both the vital anchoring end domains are present it is semifunctional. The situation is analogous to a piece of elastic, fixed at both ends. Shortening its length reduces elasticity, but it is of no use if one end is cut free. Absence of the $\mathrm{C}$ terminus gives a particularly severe phenotype. ${ }^{3}$ This hypothesis does not fit all known genotypes and loss of certain regions of the rod domain may also lead to an unstable protein. ${ }^{9}$ Many other aspects have yet to be clarified including the relation between dystrophin deficiency and both cognitive impairment and cardiac involvement, and the mechanism of calf hypertrophy. ${ }^{10}$

In DMD the absence of dystrophin gives rise to a severe secondary deficiency of all of the cell membrane dystrophin associated glycoproteins (DAGs) (fig 2). Thus, dystrophin deficiency not only removes the actin cell membrane anchor but the cell membrane-basement link is also broken. The functional interdependence of these proteins is also revealed in other forms of muscular dystrophy. In a severe autosomal recessive muscular dystrophy of childhood
(SCARMD) the phenotype and muscle histology are very similar to DMD but whilst dystrophin is present in normal amounts there is a primary deficiency of the $50 \mathrm{DAG}$, adhalin and secondary reduction in the 35DAG, hence the same links are broken (fig 2). ${ }^{6}$

\section{CONGENITAL MUSCULAR DYSTROPHY}

Congenital muscular dystrophy occurs in several forms. The Fukuyama form, frequent in Japanese patients, is a severe dystrophy associated with central nervous system anomalies that has been linked to chromosome $9 .{ }^{11}$ The commonest form in the West is solely a muscle disorder that presents with neonatal hypotonia, early contractures and a dystrophic histology including a marked increase in endomysial connective tissue. This Western variety has recently been shown to be caused by a merosin deficiency, the $M$ chain subunit of laminin and the basal lamina component that normally binds to the 156DAG (fig 2), whereas its isoform laminin A chain, is over expressed. ${ }^{12}$ No abnormalities have been demonstrated in dystrophin or dystrophin associated proteins. By contrast, in Fukuyama dystrophy, there is also normal dystrophin but a reduction the dystrophin associated protein concentrations, particularly $\beta$-dystroglycan. ${ }^{11} \mathrm{~A}$ single gene that encodes both $\alpha$-dystroglycan, the 156DAG and $\beta$-dystroglycan, the 43DAG, with expression of the transcript in brain and skeletal muscle, is a strong candidate. However, the abnormality is not as uniform as in the case of 50DAG deficient SCARMD and may vary amongst different patients with Fukuyama dystrophy.

In summary, the integrity of the sarcolemma is maintained by a protein chain that spans the membrane linking the cytoskeleton to the extracellular matrix. The dystrophin associated proteins play a pivotal role and reduction, either

Protein deficiencies in Muscular Dystrophies

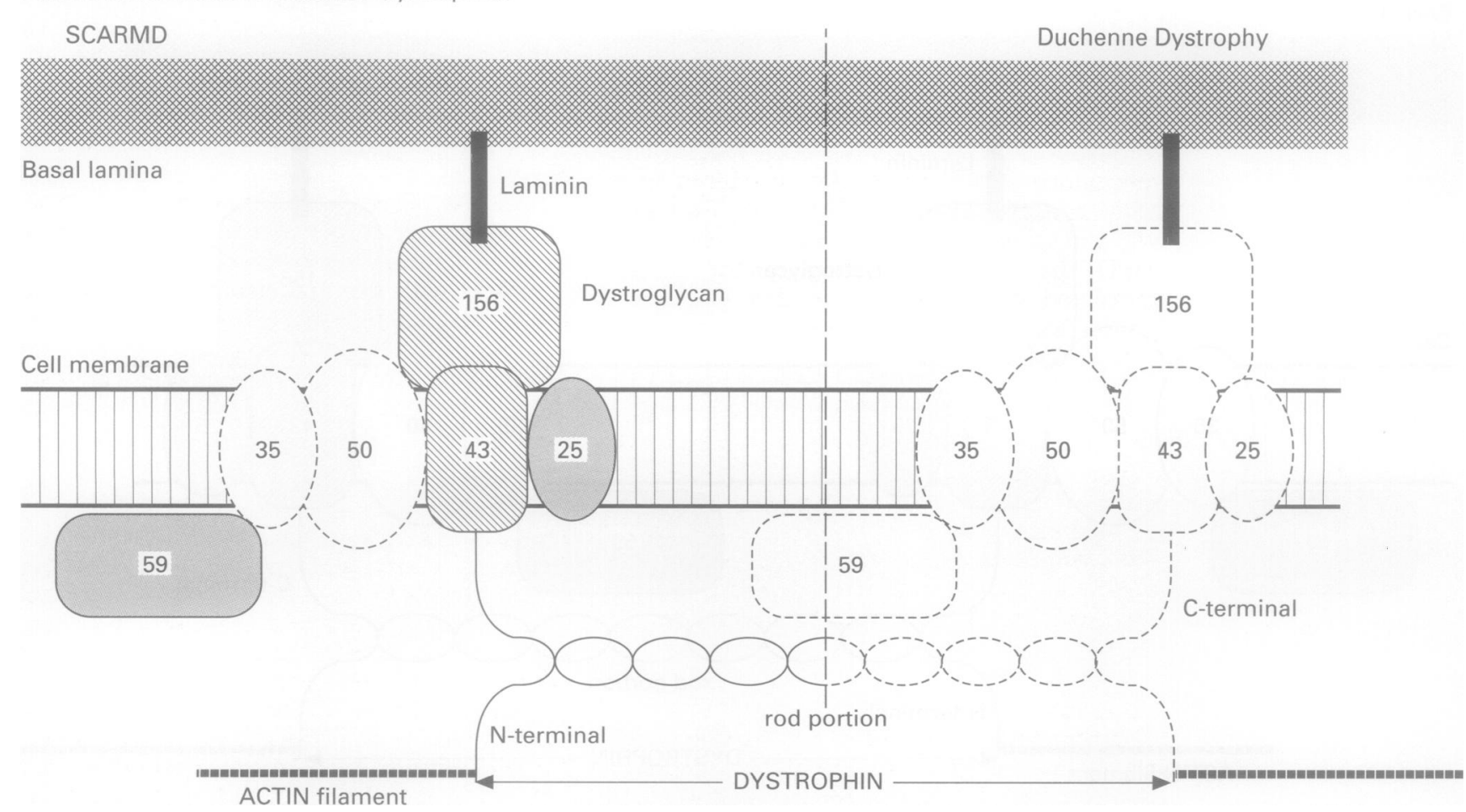

Figure 2 Dotted outlines=deficient proteins. 
primarily as in SCARMD or secondarily as in $\mathrm{DMD}$, results in a particularly severe phenotype (fig 2). Therapeutic strategies to correct the specific defects are in their infancy but approaches include myoblast transplant and the introduction of new genetic material into somatic cells using viral vectors or direct DNA transfer. ${ }^{13}$

The molecular basis for other forms of muscular dystrophy, including the heterogeneous limb girdle dystrophies and facioscapulohumeral dystrophy has yet to be uncovered, but the genetic location for the latter and for a large number of neuromuscular disorders has already been isolated. ${ }^{14} 15$

\section{MYOTONIC DYSTROPHY}

The wide phenotypic range of myotonic dystrophy (MyD) extends from profound neonatal respiratory distress to cataracts and minimal muscular weakness in middle age. The identification of a new mechanism of mutation in man resolved a longstanding debate and confirmed that the earlier appearance of MyD with increasing severity in successive generations was a true biological phenomenon (anticipation), not simply the result of observer bias. This anticipation is attributable to an unstable DNA triplet repeat sequence, in which an increasing number of CTG triplet repeats correlates with increasing severity of the phenotype. ${ }^{16}$ Clinical manifestation is associated with at least a doubling of the normal repeat range. Families show anticipation in both the clinical and molecular sense as the sequence is expanded in most children who inherit the mutation. Furthermore, all other diseases in which anticipation has been recognised, namely Fragile X syndrome, X linked spinal muscular atrophy, Huntington's chorea and spinocerebellar ataxia type 1 , and dentatorubralpallidoluysian atrophy, the same mutational mechanism has been demonstrated. ${ }^{17}$ In MyD there is some overlap in the size of expansion between moderately severe juvenile onset cases and milder adult onset individuals, but no overlap exists between the minimally affected individual and the congenital form. The mechanism of exclusive maternal inheritance of the congenital form is still not fully explained and an in utero effect is still postulated. In MyD the expansion occurs in the $3^{\prime}$ untranslated region of a gene that encodes a protein kinase, DM-PK. Expression is maximal in cardiac muscle but it is also present in skeletal and smooth muscle and brain. ${ }^{18}$ Immunofluorescence studies of skeletal muscle show a cell membrane localisation that is particularly strong at the $\mathrm{N}-\mathrm{M}$ junction. The mechanism whereby expansion of an untranslated region of the gene has such far reaching effects is still unknown, but initial transgenic mouse experiments suggest that overexpression of DMPK modifies other cell membrane proteins. ${ }^{19}$

\section{Congenital myopathies}

The congenital myopathies are a group of relatively non-progressive, heritable disorders characterised by distinctive cytoarchitectural abnormalities upon which nomenclature is based. A long held concept of failure of mat- uration is supported by expression of fetal proteins in some cases, but greater diversity has become apparent with the identification of genetic defects affecting different cytoskeletal components. Unlike the dystrophies these abnormalities are not associated with muscle cell necrosis.

Central core disease is an autosomal dominant myopathy in which a minority of patients exhibit susceptibility to malignant hyperthermia $(\mathrm{MH})$. The $\mathrm{MH}$ susceptibility gene has been localised to chromosome 19q12-13.2 and corresponds to the ryanodine receptor, a calcium channel receptor. Mutations in this gene are responsible for $\mathrm{MH}$ susceptibility. Linkage studies in families with central core disease localised to the same region on chromosome $19 q 13 \cdot 1$ and showed that these are allelic genes. ${ }^{20}$ However, there is genetic heterogeneity in these two diseases and variable phenotypic expression occurs even within a family. The number of cores, the central cylindrical disturbance of the myofibrils, increases with age and the percentage of type 1 fibres increases but the molecular mechanisms underlying these morphological changes still await discovery.

Nemaline myopathy is also included in the category with distinctive fine structural abnormalities. It is almost always an autosomal recessive disorder, but there is also a separate autosomal recessive form characterised by intranuclear rods and a rare autosomal dominant form. The latter has very recently been shown to be caused by a mutation in the actin binding site of tropomyosin 3, the gene for which is located on chromosome 1q21-23 (Laing NG, unpublished observations). Other tropomyosins are potential candidates for the autosomal recessive types.

Myotubular myopathy is an X linked inherited disorder with profound hypotonia in which a morphological resemblance to fetal myotubes has long suggested a maturational arrest. The myotube-like fibres retain fetal isoforms of myosin and also express desmin and vimentin, a myotube characteristic. These fetal characteristics are not found in morphologically similar, but milder, autosomal dominant conditions. ${ }^{21}$

The list of congenital myopathies is gradually being enlarged with the addition of rare, unusual familial myopathies. ${ }^{21}$ In all of these disorders with unusual structural changes an orchestrated approach combining immunocytochemistry to examine muscle proteins in situ and molecular genetics to examine candidate genes may eventually unravel the pathogenesis.

\section{Mitochondrial cytopathies}

In recent years mutations of mitochondrial DNA (mtDNA) have been recognised as the basis of a large group of multisystem disorders, with diverse clinical manifestations, but in which encephalomyopathies often predominate. ${ }^{22}$ Brain and muscle are particularly susceptible to mitochondrial dysfunction because of their high oxidative metabolic activity. The majority of mitochondrial proteins are transcribed from nuclear DNA, but the mitochondrion also contains its own, unique, small 
circular DNA which encodes the remainder and directs synthesis within the mitochondrion. The mtDNA contains genes that encode several vital subunits of complexes I, III, IV, and V of the respiratory chain. Sequencing of the entire mitochondrial genome has identified many pathogenic mutations, including point mutations and large scale deletions or duplications. ${ }^{23}$ Mitochondria within the fertilised egg are almost exclusively derived from the ovum and diseases due to point mutations of the mitochondrial genome often show strict maternal inheritance. Diseases that result from a large deletion or duplication are usually sporadic, possibly because such large scale rearrangements of mtDNA affect viability of the ovum and are not transmitted.

The majority of the proteins of the respiratory chain enzymes are nuclear encoded and are synthesised as precursors in the cytoplasm before transportation into the mitochondrion for final assembly with mtDNA transcribed proteins. Nuclear DNA mutations may interfere at various points in the complex intergenomic communication. ${ }^{24}$ Mitochondrial myopathies with Mendelian inheritance may show generalised reduction in mtDNA encoded enzymes because mtDNA replication is under nuclear control. Analysis of mtDNA usually reveals deletions at multiple sites involving a large portion of the genome. ${ }^{25}$

Within a wide spectrum, encompassing rapidly fatal encephalomyopathies of infancy to late adult onset of ptosis and mild myopathy, several distinctive clinical syndromes are associated with mitochondrial defects. MELAS (mitochondrial encephalomyopathy, lactic acidosis and stroke-like episodes) and MERRF (myoclonic epilepsy and ragged red fibres) are attributable to different point mutations in mitochondrial transfer RNA genes. ${ }^{2326}$ Chronic progressive external ophthalmoplegia and ragged red fibre on muscle biopsy and ophthalmoplegia plus the Kearns-Sayre syndrome are strongly correlated with large deletions or duplications of mtDNA. Nevertheless, there is clinical overlap between these syndromes and wide variation in the severity of the phenotype even amongst family members. ${ }^{27}$ The overlap occurs because these mutations all produce multiple partial defects of mtDNA dependent respiratory complexes. Variation is largely determined by two factors, the tissue distribution and proportion of mutant mtDNA. During embryogenesis, defective and normal mitochondria are randomly dispersed with resulting heteroplasmy in many tissues. Cells may contain variable proportions of mutant and wildtype mtDNA but as every cell contains numerous mitochondria and many copies of mtDNA, the mutant genome is not expressed until the level exceeds a certain threshold. In the permanent cells of mature skeletal and cardiac muscle and neurons, cells with a large number of abnormal mitochondria cannot be eliminated and replaced through cell division. Furthermore, smaller mutant mtDNAs may have a replicative advantage and therefore increase with age. Thus, presentation of mitochondrial disorders is frequently delayed until adult life and even old age. Numerous clinical mitochondrial syndromes have now been described and new ones are continually being docu- mented-for example, diabetes and deafness with maternal inheritance have been described recently, hence the possibility should be entertained in anyone with an unexplained progressive multisystem disorder. ${ }^{28}$

The microscopic hallmark of a mitochondrial myopathy is the ragged red fibre in skeletal muscle biopsy specimens stained with Gomori's trichrome. The appearance is due to large subsarcolemmal aggregates of mitochondria in which electron microscopy reveals abnormal structure, either excessive and whorled cristae or crystalline inclusions. Ragged red fibres are frequently cytochrome oxidase negative and are the visible evidence of diminished mtDNA translation. Thus, they are almost universally associated with large mtDNA deletions and frequently with point mutations of mitochondrial transfer RNA genes, but do not occur with mtDNA point mutations of individual messenger RNA genes - for example, Leber's optic atrophy, and only occur with nuclear mutations that cause mtDNA depletion.

Muscle biopsy has been the traditional first line approach to diagnosis. However, mtDNA point deletions can be readily detected by PCR on a peripheral blood sample and this is a preferable initial screening test in patients with a suggestive clinical picture. Large deletions are not readily detected in this way and muscle biopsy is required. ${ }^{29}$ If there are no ragged red fibres and the mitochondrial genome analysis does not pick up mutations, biochemical analysis of the snap frozen muscle biopsy specimen may confirm a respiratory chain defect.

\section{Inflammatory myopathies}

Although historically the idiopathic inflammatory myopathies have been regarded as overlapping disorders, clear differences have emerged in the immunopathogenesis of polymyositis (PM) and dermatomyositis (DM). The former is characterised by cytotoxic $T$ cell invasion and destruction of intact muscle fibres that express HLA class I antigens, whereas in DM the intramuscular microvasculature is the primary target of a humoral immune response. $^{30}$ In DM circulating endothelial cell antibodies have been demonstrated and the complement cascade is activated in the walls of intramuscular capillaries. Formation of the terminal component, membrane attack complex causes capillary lysis and muscle fibre necrosis due to ischaemia. The triggers of these autoimmune disorders are still largely a matter of conjecture. Viruses have long been regarded as likely candidates. The association of HIV with a myopathy indistinguishable from PM supports this hypothesis, but PCR studies to detect viral genomic material in muscle biopsy specimens from patients with the acquired immunodeficiency syndrome (AIDS) and for likely viral initiators, such as Coxsackie, Epstein-Barr virus, mumps and adenovirus, in non-AIDS cases have been unsuccessful. The failure to identify viral DNA within muscle fibres, despite a strong causal association in patients with AIDS, suggests that a preliminary viral infection can expose or modify self antigens, but subsequently there is neither persistent nor latent infection. Other environmental triggers, such as drugs, appear to be 
responsible in occasional cases and the link between DM or PM and malignancy, often overstated but nevertheless probably genuine, invokes a tumour cell-muscle fibre antigenic cross reaction.

Inclusion body myositis has been recognised in recent years as a not uncommon, slowly progressive, inflammatory myopathy of older people that fails to respond to steroid therapy. Much diagnostic confusion has arisen because in many instances the diagnosis has rested solely upon histological recognition of rimmed vacuoles irrespective of the clinical picture and other findings, including inflammation. Electron microscopy shows that the rimmed vacuoles are large autophagic vacuoles accompanied by distinctive cytoplasmic and intranuclear filaments. These filaments are always associated with degenerating organelles. Occasional rimmed vacuoles occur in diverse neuromuscular disorders, but they are particularly numerous in a sporadic inflammatory disorder of older patients, justifiably referred to as inclusion body myositis, and also in certain hereditary disorders devoid of inflammation and thus better termed inclusion body myopathies. ${ }^{31}$ Apart from inflammation, the changes in hereditary myopathies and the sporadic disease are identical. Furthermore, the degree of inflammation does not correlate with disease severity and it seems likely that inflammation represents a secondary phenomenon and not the primary disease mechanism.

The filaments closely resemble the paired helical filaments of Alzheimer's disease. Similarity with Alzheimer's disease is heightened by the demonstration of small deposits of Congo red positive amyloid in vacuoles alongside an accumulation of many proteins that are found in the Alzheimer brain, including $\beta$-amyloid protein, ubiquitin, $\alpha$-1-antichymotrypsin, apolipoprotein $\mathrm{E}$ and phosphorylated $\tau .{ }^{3132}$ Only $\tau$, ubiquitin and apolipoprotein $E$ are localised to the filaments. Increased synthesis and accumulation of $\beta$ amyloid precursor protein, the nicotinic acetylcholine receptor and the cellular isoform of prion protein has also been demonstrated. These three proteins are all present in normal muscle but concentrated at the neuromuscular junction. Affinity with Alzheimer's disease is tantalising and suggests parallel metabolic derangements are responsible for the two degenerative diseases. The detection of a single stranded, seemingly non-viral, DNA binding protein in myonuclei and rimmed vacuoles in inclusion body muscle diseases has lead to the proposal that nuclear damage is the fundamental event. ${ }^{33}$

The accelerating progress of a multidisciplinary approach to neuromuscular diseases continues to elucidate pathogeneses thereby paving the way for rapid diagnostic tests and future development of specific therapeutic strategies in disorders which hitherto have been poorly understood, frequently incorrectly diagnosed and largely incurable.

1 Monaco AP, Neve RL, Colletti-Feener C, Bertelson CJ, Kurnit DM, Kunkel LM. Isolation of candidate cDNAs from portions of the Duchenne muscular dystrophy gene. Nature 1986;316:842-5.

2 Hoffman E, Brown RH. Dystrophin: The product of the Duchenne muscular dystrophy locus. Cell 1987;51:991928.

3 Matsumura K, Campbell KP. Dystrophin-glycoprotein complex: its role in the pathogenesis of muscular dystrophies. Muscle Nerve 1994;17:2-15.

4 Campbell KP, Sunada Y. Dystroglycan: a novel merosin receptor in the molecular pathogenesis of muscular dystrophy. Muscle Nerve 1994;17:S12.

5 Minetti C, Beltame F, Marcenaro G, Bonilla E. Dystrophin at the plasma membrane of human muscle fibres shows at the plasma membrane of human muscle fibres shows
a constameric localisation. Neuromuscul Disord 1992;2: a constam

6 Matsumura K, Campbell KP. Deficiency of dystrophinassociated proteins: a common mechanism leading to muscle cell neurosis in severe childhood muscular dystrophies. Neuromuscul Disord 1993;3:109-18.

7 Fassati A, Tedeschi S, Bordoni A, Amboni P, Curcio C, Bresolin N, et al. Rapid diagnosis of deletion carriers of Duchenne and Becker muscular dystrophies. Lancet 1994; 344:302-3.

8 Monaco AP, Bertelson CJ, Liechti-Gallati S, Moser H, Kunkel L. An explanation for the phenotypic differences between patients bearing partial deletions of the DMD between patients bearing partial
locus. Gernomics 1988;2:90-5.

9 Matsumura K, Ohlendieck K, Ionasescu VV, Tome FMS, Nonaka I, Burghes AHM, et al. The role of the dystrophinglycoprotein complex in the molecular pathogenesis of muscular dystrophies. Neuromuscul Disond 1994;3:533-5.

10 Emery AEH. Some unanswered questions in Duchenne muscular dystrophy. Neuromuscul Disord 1994;4:301-3.

11 Matsumura K, Nonaka I, Campbell KP. Anormal expression of dystrophin-associated proteins in Fukuyama-type congenital muscular dystrophy. Lancet 1993;341:521-2.

12 Tome FS, Evangelista T, Leclerc A. Congenital muscular dystrophy with merosin deficiency. CR Acad Sci Paris, Life Sciences, Genetics: 1994;317:351-7.

13 Goss F. Gene Therapy: present situation and future pro-

spects. Neuromuscul Disord 1992;2:75-84.
14 Wijmenga C, Frants RR, Hewitt JE, van Deutekom JCT, van Geel M, Wright TJ, et al. Molecular genetics of facioscapulohumeral dystrophy. Neuromuscul Disord 1993; 3:487-91.

15 Neuromuscular disorders: gene location. Neuromuscul Disord 1994;4:393-5.

16 Brook JD, McCurrach ME, Harley HG. Molecular basis of myotonic dystrophy: expansion of a trinucleotide (CTG)
repeat at the $3^{\prime}$ end of a transcript encoding a protein kinase family member. Cell 1992;68:799-808.

17 Caskey CT. Trinucleotide repeat associated diseases. Muscle Nerve 1994;17:S11.

18 Strong PN, Brewster BS, Sewry CA, Jeal S, Wilson LA. Expression of the myotonic dystrophy end product. Muscle Nerve 1994;17:S13.

19 Jansen G, van der Ven PJ, ter Riele H, Groenen PBW. Animal models mimicking myotonic dystrophy over- and underexpression. Muscle Nerve 1994;17:S13.

20 Quane KA, Healy JM, Keating KE, Manning BM, Couch FJ, Palmucci LM, et al. Mutations in the ryanodine receptor gene in central core disease and malignant hyperthermia. Nature Genet 1993;5:51-5.

21 Bodensteiner JB. Congenital Myopathies. Muscle Nerve 1994;17:131-44.

22 DiMauro S, Moraes CT. Mitochondrial encephalomyopathies. Arch Neurl 1993;50:1197-208.

23 Zeviani MCA. Defects of mitochondrial DNA. Brain Pathol 1992;2:121-32.

24 Zeviani M, Di Donato S. Neurological disorders due to mutations of the mitochondrial genome. Neuromuscul Disord 1991;1:165-72.

25 Ricci E, Carol TM, Serenella S, Tonali P, Bonilla E, DiMauro S. Disorders associated with depletion of mitochondrial DNA. Brain Pathol 1992;2:141-7.

26 Koo B, Becker LE, Chuang S, Merante F, Robinson BH, MacGregor D, et al. Mitochondrial encephalomyopathy, lactic acidosis, stroke-like episodes (MELAS): clinical, radiological, pathological and genetic observations. Ann Neurol 1993;34:25-32.

27 Hirano M, Ricci E, Koenigsberger R, Defendini R, Pavladkis S, DeVivo DC, et al. MELAS: An original case and clinical $S$, DeVivo DC, et al. MELAS: An original case and clinical
criteria for diagnosis. Neuromuscul Disond 1992;2:125-35. 28 Balinger SW, Shoffner JM, Hedaya EV, Trounce I, Polak MA, Koontz DA, et al. Maternally transmitted diabetes and deafness associated with a $10.4 \mathrm{~kb}$ mitochondrial DNA deletion. Nature Genet 1992;4:11-15.

29 Harding AE. The DNA laboratory and neurological practice. Neurol Neurosurg Psychiatry 1993;56:229-33.

30 Carpenter S, Karpati G. The pathogenesis of specific inflammatory myopathies. Brain Pathol 1992;2:13-19.

31 Askanas V, Engel WK. New advances in inclusion body myositis. Curr Opinion Rheumatol 1993;5:732-41.

32 Askanas V, Mirabella M, Engel WK, Alvarez RB, Weisgraber KH. Apolipoprotein E immunoreactive deposits

33 Nalbantoglu J, Karpati G, Carpenter S. Conspicuous accumulation of a single stranded DNA binding protein in cumulation of a single stranded DNA binding protein in Pathol 1994;144:874-882. 